\title{
CÁLCULO DE DISPONIBILIDADE EM SIMULADOR DE PROCESSOS
}

\author{
A. M. AVELAR ${ }^{1}$, F. W. TAVARES 2
}

${ }^{1}$ Marinha do Brasil, Diretoria de Abastecimento da Marinha, Departamento Técnico

${ }^{2}$ Universidade Federal do Rio de Janeiro, Escola de Química, Departamento de Engenharia Química

E-mail para contato: aolbr_mail@yahoo.com.br

\begin{abstract}
RESUMO - O cálculo da exergia em correntes materiais e de energia foi implementado no simulador de processos Aspen HYSYS®. Para cada tipo de operação ou processo unitário foram programados os cálculos de irreversibilidade e de eficiência. As rotinas de programação foram validadas a partir de comparações com resultados encontrados na literatura. Nos exemplos analisados, critérios termoeconômicos e ambientais foram considerados, e os resultados demonstraram que a eficiência racional, diretamente relacionada ao índice de sustentabilidade do processo, consegue lidar com a ordem de grandeza da escala completa de disponibilidade. Além disso, atribuição de custos exergéticos na formulação do custo real dos produtos pode ser utilizada para mensurar, de maneira extensiva, o impacto ambiental e avaliar a viabilidade econômica de tecnologias sustentáveis.
\end{abstract}

\section{INTRODUÇÃO}

Entende-se por exergia de uma dada corrente material, o trabalho máximo que se pode obter levando-se uma dada corrente ao estado de equilíbrio com o ambiente, por um processo reversível. A disponibilidade de energia, ou simplesmente exergia, é destruída devido as irreversibilidades dos processos. A análise de disponibilidade, ou exergética, permite a avaliação de processos, sob o ponto de vista da segunda lei da termodinâmica, baseada em uma escala cujo estado de referência é o meio ambiente (Kotas., 1985).

A maioria dos simuladores de processos existentes no mercado: Aspen HYSYS®, Aspen Plus ${ }^{\circledR}$, ProII ${ }^{\circledR}$, EMSO/VRTHERM, não realizam o cálculo de disponibilidade em seus pacotes termodinâmicos. Diversos trabalhos iniciaram as implementações do cálculo da exergia em simuladores de processo (Montelongo-Luna, 2007; Abdollahi-Demneh et al., 2011; Ghannadzadeh et al., 2012). O trabalho de Ghannadzadeh et al. (2012) faz uma revisão da literatura, comparando as implementações em simuladores de processos. O presente trabalho objetiva estender a implementação de Abdollahi-Demneh et al. (2011), aplicando também o cálculo da exergia de correntes de energia, cálculo da eficiência racional de operações unitárias de acordo com Ghannadzadeh et al. (2012), avaliação termoeconômica e ambiental de acordo com Valero et al. (1993) e Rosen et al. (2008). 


\section{IMPLEMENTAÇÃO}

O procedimento proposto por Abdollahi-Demneh et al., (2011) consiste em um método direto, sem repetições de programação, para o cálculo da exergia de uma corrente de processo, implementada através de código em Visual Basic no programa simulador de processos Aspen HYSYS®.

A escala de disponibilidade utiliza como referência um estado denominado de "morto", haja vista que, a partir dele, não é possível produzir trabalho. O estado morto de referência aqui adotado, foi sugerido por Szargut et al. (1988), conforme descrito na Tabela 1, pois, também fora utilizado em diversos trabalhos recentes (Montelongo-Luna, 2007; AbdollahiDemneh et al., 2011; Ghannadzadeh et al., 2012).

Tabela 1 - Estado morto de referência proposto por Szargut et al. (1988).

\begin{tabular}{|c|c|}
\hline Temperatura $\left(\mathrm{T}_{00}\right)$ & $298,15 \mathrm{~K}$ \\
\hline Pressão $\left(\mathrm{P}_{00}\right)$ & $99312 \mathrm{~Pa}$ \\
\hline Componente & Pressão parcial $(\mathrm{Pa})$ \\
\hline $\mathrm{N}_{2}$ & 75780 \\
\hline $\mathrm{O}_{2}$ & 20390 \\
\hline $\mathrm{CO}_{2}$ & 33,5 \\
\hline $\mathrm{H}_{2} \mathrm{O}$ & 2200 \\
\hline $\mathrm{D}_{2} \mathrm{O}$ & 0,342 \\
\hline $\mathrm{Ar}$ & 906 \\
\hline $\mathrm{Xe}$ & 0,0087 \\
\hline $\mathrm{Ne}$ & 1,77 \\
\hline $\mathrm{Kr}$ & 0,097 \\
\hline $\mathrm{He}$ & 0,485 \\
\hline
\end{tabular}

A exergia física de uma corrente material corresponde ao trabalho obtido levando-se a corrente do seu estado inicial de temperatura e pressão até as condições de temperatura e pressão do ambiente, por meio de um processo reversível. Corresponde a Etapa 1 da Figura 1.

A exergia química de uma corrente material corresponde ao trabalho obtido pela transformação química da composição de uma unidade de massa da corrente, nas condições de temperatura e pressão do ambiente, até o equilíbrio completo com o meio ambiente, em termos de composição inclusive. O procedimento proposto por Abdollahi-Demneh et al. (2011) utiliza o método proposto por Szargut et al. (1988) para o cálculo da exergia química, sendo divido em quatro etapas, aqui nomeadas de Etapas 2, 3, 4 e 5.

$\mathrm{Na}$ Etapa 2, todas as substâncias contidas na mistura nas condições ambiente de temperatura e pressão $\left(\mathrm{T}_{0}\right.$ e $\left.\mathrm{P}_{0}\right)$ são conduzidas ao seu estado de gás ideal e substância pura, 
na mesma condição de temperatura $\left(\mathrm{T}_{0}\right)$ e na condição de pressão de referência do simulador $\left(\mathrm{P}_{0 \mathrm{n}}=101325 \mathrm{~Pa}\right)$. Os autores Abdollahi-Demneh et al. (2011) utilizam a expressão do calor específico dos componentes ideais puros, implementada no próprio programa para cálculo da entalpia e entropia dos componentes.

Na Etapa 3, todas as substância puras; exceto aquelas que não possuem espécie química de referência, como por exemplo, hidrocarbonetos halogenados; são convertidas por reações reversíveis, conforme a Equação 1, na temperatura ambiente $\left(\mathrm{T}_{0}\right)$ e na pressão de referência do simulador $\left(\mathrm{P}_{0 \mathrm{n}}=101325 \mathrm{~Pa}\right)$, dando origem as espécies de referência do estado morto.

$$
\begin{aligned}
& \mathrm{X}_{\mathrm{i}}+v_{\mathrm{i} 1} \mathrm{O}_{2} \leftrightarrow v_{\mathrm{i} 2} \mathrm{CO}_{2}+v_{\mathrm{i} 3} \mathrm{H}_{2} \mathrm{O}+ \\
& v_{i 4} \mathrm{~N}_{2}+v_{\mathrm{i} 5} \mathrm{Ar}+v_{\mathrm{i} 6} \mathrm{Xe}+v_{\mathrm{i} 7} \mathrm{Ne}+v_{\mathrm{i} 8} \mathrm{Kr}+v_{\mathrm{i} 9} \mathrm{He}+v_{\mathrm{i} 10} \mathrm{D}_{2} \mathrm{O}+v_{\mathrm{i} 11} \mathrm{~S}_{\text {(sólido) }}
\end{aligned}
$$

$\mathrm{Na}$ Etapa 4, as pressões parciais das substâncias de referência são alteradas da condição de substância pura na pressão de referência do simulador $\left(\mathrm{P}_{0 \mathrm{n}}=101325 \mathrm{~Pa}\right)$, para as pressões parciais do estado morto de referência da Tabela 1, de maneira reversivel e isotérmica, uma vez que a temperatura do estado morto de referência $\left(\mathrm{T}_{00}=298,15 \mathrm{~K}\right)$ definido por Szargut, et al. (1988) é a mesma temperatura ambiente $\left(\mathrm{T}_{0}=298,15 \mathrm{~K}\right)$. É válido ressaltar que a pressão total do estado morto de referência é $\mathrm{P}_{00}=99312 \mathrm{~Pa}$, sendo necessário uma etapa posterior para corrigir a exergia ao estado morto real. Para o caso específico do enxofre sólido rômbico, recomenda-se o cálculo deste componente com base no íon sulfato dissolvido na água do mar, sendo $\Delta \mathrm{B}_{\mathrm{S}}=609,6 \mathrm{~J} / \mathrm{kgmol}$. (Abdollahi-Demneh et al. 2011)

Na Etapa 5, as pressões parciais das espécies de referência são alteradas dos valores do estado morto de referência, contidos na Tabela 1 , que somados resultam na $\mathrm{P}_{00}=99312 \mathrm{~Pa}$, para a pressão do ambiente $\mathrm{P}_{0}=101325 \mathrm{~Pa}$, de maneira reversivel e isotérmica. Assume-se que a concentração das espécies é a mesma em ambos os estados. Agrupando-se todas as etapas, conforme ilustrado na Figura 1, obtêm-se a Equação 3 para o cálculo da exergia de uma corrente de processo.

No caso de correntes de energia, o cálculo é realizado de forma diferente para correntes de calor e trabalho. Para uma corrente de trabalho, o valor da exergia é igual à potência da corrente. Por outro lado, para uma corrente de calor, a exergia é calculada aplicando uma máquina de Carnot, cuja eficiência é dada pela Equação 2:

$$
\eta=1-\frac{\mathrm{T}_{0}}{\overline{\mathrm{T}}}=1-\frac{\mathrm{T}_{0} \cdot \Delta \mathrm{S}_{\mathrm{UTILIDADE}}}{\Delta \mathrm{H}_{\mathrm{UTILIDADE}}}
$$




\begin{tabular}{|c|c|c|c|c|c|c|}
\hline 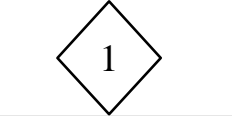 & ETAPA 1 & AMBIENTE & ETAPA 2 & $\begin{array}{c}\text { COMPONENTES } \\
\text { PUROS }\end{array}$ & ETAPA 3 & $\begin{array}{c}\text { DE REFERÊNCIA } \\
\text { PUROS }\end{array}$ \\
\hline $\begin{array}{llll}\mathrm{T}_{1} & \mathrm{P}_{1} & \underline{\mathrm{z}_{1}} \\
\end{array}$ & & $\begin{array}{lll}\mathrm{T}_{0} & \mathrm{P}_{0} & \underline{\mathrm{z}_{1}} \\
\end{array}$ & & $\mathrm{~T}_{0} \quad \mathrm{P}_{0 \mathrm{n}}$ & & $\begin{array}{ll}\mathrm{T}_{0} \quad \mathrm{P}_{0 \mathrm{n}} \\
\end{array}$ \\
\hline
\end{tabular}

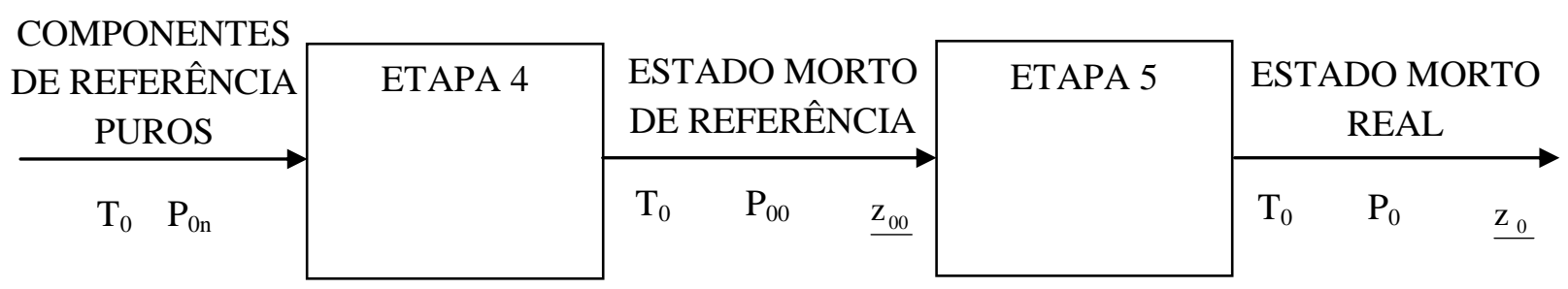

Figura 1 - Conjunto de transformações com todas etapas do procedimento para o cálculo da exergia de corrente de processo. Adaptado de Abdollahi-Demneh et al. (2011).

$$
\begin{aligned}
& B=\left(H\left(T_{1}, P_{1}, \underline{z_{1}}\right)-H\left(T_{0}, P_{0}, \underline{z_{1}}\right)\right)-T_{0} \cdot\left(S\left(T_{1}, P_{1}, \underline{z_{1}}\right)-S\left(T_{0}, P_{0}, \underline{z_{1}}\right)\right)+ \\
& \left(H\left(T_{0}, P_{0}, \underline{z_{1}}\right)-H^{i d}\left(T_{0}, \underline{z_{1}}\right)\right)-T_{0} \cdot\left(S\left(T_{0}, P_{0}, \underline{z_{1}}\right)-S^{i d}\left(T_{0}, P_{0 n}, \underline{z_{1}}\right)+\frac{R}{M M\left(\underline{z_{1}}\right)} \cdot \sum_{i=1}^{n} z_{i} \cdot \ln \left(z_{i}\right)\right)+ \\
& \frac{1}{M M\left(\underline{\left.z_{1}\right)}\right.} \cdot \sum_{i=1}^{n} z_{i} \sum_{j=1}^{10} v_{i j} \cdot\left(R \cdot T_{0} \cdot \ln \left(\frac{\sum_{j=1}^{10} P_{r e f j}}{P_{0}} \cdot \frac{P_{0 n}}{P_{r e f j}}\right)+G_{f, j}+v_{i 11} \cdot \Delta B_{S}\right)
\end{aligned}
$$




\section{RESULTADOS E DISCUSSÕES}

Os exemplos numéricos apresentados por Abdollahi-Demneh et al. (2011) foram simulados, utilizando o mesmo modelo termodinâmico adotado pelo autor (SRK - SoaveRedlich-Kwong), e os resultados foram validados por comparação. Os resultados obtidos referentes à Corrente de gás natural (Temperatura 303,15 K, Pressão 6200 kPa e Vazão molar $500 \mathrm{kgmol} / \mathrm{h}$ ) encontram-se na Tabela 3, cuja composição encontra-se descrita na Tabela 2,. Outra validação foi conduzida para a exergia química de diversas substâncias, nas condições de 298,15 K e 1 bar. Estes dados encontram-se apresentados em diversos trabalhos científicos. (Lora et al., 2004), (Gharagheizi et al., 2007) e (Zanchini et al., 2009). Os resultados obtidos para algumas substâncias encontram-se na Tabela 4.

Os resultados obtidos para exergia química e física de correntes de gás natural estão de acordo com os publicados por Abdollahi-Demneh et al. (2011). Verifica-se também que os dados obtidos para exergia química padrão a $298 \mathrm{~K}$ e 1 bar, estão de acordo com os obtidos nos demais trabalhos da literatura. Ambas as verificações configuram uma validação para as rotinas programadas no Aspen HYSYS®. Uma vez validada a implementação, foram escolhidos dois exemplos de processos para utilizar a implementação sendo: (1) coluna despropanizadora e (2) planta térmica simplificada.

Tabela 2 - Composição da corrente de gás natural. Adaptado de Abdollahi-Demneh et al. (2011)

\begin{tabular}{cc}
\hline Componente & Fração molar \\
\hline $\mathrm{N}_{2}$ & 0,001 \\
$\mathrm{H}_{2} \mathrm{~S}$ & 0,01544 \\
$\mathrm{CO}_{2}$ & 0,02835 \\
$\mathrm{CH}_{4}$ & 0,8982 \\
$\mathrm{C}_{2} \mathrm{H}_{6}$ & 0,03098 \\
$\mathrm{C}_{3} \mathrm{H}_{8}$ & 0,01479 \\
$\mathrm{i}-\mathrm{C}_{4} \mathrm{H}_{10}$ & 0,0059 \\
$\mathrm{n}-\mathrm{C}_{4} \mathrm{H}_{10}$ & 0,003 \\
$\mathrm{i}-\mathrm{C}_{5} \mathrm{H}_{10}$ & 0,001 \\
$\mathrm{n}-\mathrm{C}_{5} \mathrm{H}_{10}$ & 0,0005 \\
$\mathrm{H}_{2} \mathrm{O}$ & 0,00086 \\
\hline
\end{tabular}

Tabela 3 - Comparação entre os resultados obtidos para a Corrente de gás natural.

\begin{tabular}{cccc}
\hline Origem & $\begin{array}{c}\text { Exergia Física } \\
(\mathbf{k W})\end{array}$ & $\begin{array}{c}\text { Exergia Química } \\
(\mathbf{k W})\end{array}$ & $\begin{array}{c}\text { Exergia } \\
(\mathbf{k W})\end{array}$ \\
\hline Abdollahi-Demneh et al. $(2011)$ & 1376,5 & 118437,1 & 119813,6 \\
Presente trabalho & 1372,5 & 118558,7 & 119931,2 \\
\hline
\end{tabular}


Tabela 4 - Exergia Química Molar de alguns componentes a $298 \mathrm{~K}$ e 1 bar. (Modelo termodinâmico utilizado no Aspen HYSYS®: SRK - Soave-Redlich-Kwong)

\begin{tabular}{cccccc}
\hline & \multicolumn{5}{c}{$\begin{array}{c}\text { Exergia Química } \\
\text { (kJ/kmol) }\end{array}$} \\
\cline { 2 - 6 } Componente & Kotas & Gharagheizi et & Zanchini et & Lora et al. & Presente \\
& $\mathbf{( 1 9 8 5 )}$ & al. $\mathbf{( 2 0 0 7 )}$ & al. $(\mathbf{2 0 0 9 )}$ & $\begin{array}{c}\text { P04) } \\
\text { trabalho }\end{array}$ \\
\hline $\mathrm{H}_{2}$ & 238490 & - & 235358 & 235428 & 235317 \\
$\mathrm{CH}_{4}$ & 836510 & 837479 & 830494 & 830891 & 830619 \\
$\mathrm{C}_{2} \mathrm{H}_{6}$ & 1504360 & 1493749 & 1494690 & 1495052 & 1493320 \\
$\mathrm{C}_{3} \mathrm{H}_{8}$ & 2163190 & 2159909 & 2147850 & 2150830 & 2148343 \\
\hline
\end{tabular}

No exemplo da coluna despropanizadora, verificou-se o efeito da razão de refluxo na atribuição dos custos exergéticos das correntes de topo e fundo. $\mathrm{O}$ aumento da razão de refluxo proporciona que a separação seja efetuada como um menor número de estágios. Entretanto, há um aumento no dispêndio exergético principalmente na corrente de vapor do refervedor e na irreversibilidade da operação, cuja consequência é o aumento do custo exergéticos dos produtos. Concatenando os resultados da análise termoeconômica com o cálculo do índice de sustentabilidade, proposto por Rosen et al. (2008), é possível observar, conforme a Figura 2, que quanto maior o custo exergético do produto de topo, menor é o índice de sustentabilidade do processo. Este comportamento inverso está de acordo com as premissas estabelecidas (Rosen et al., 2008).

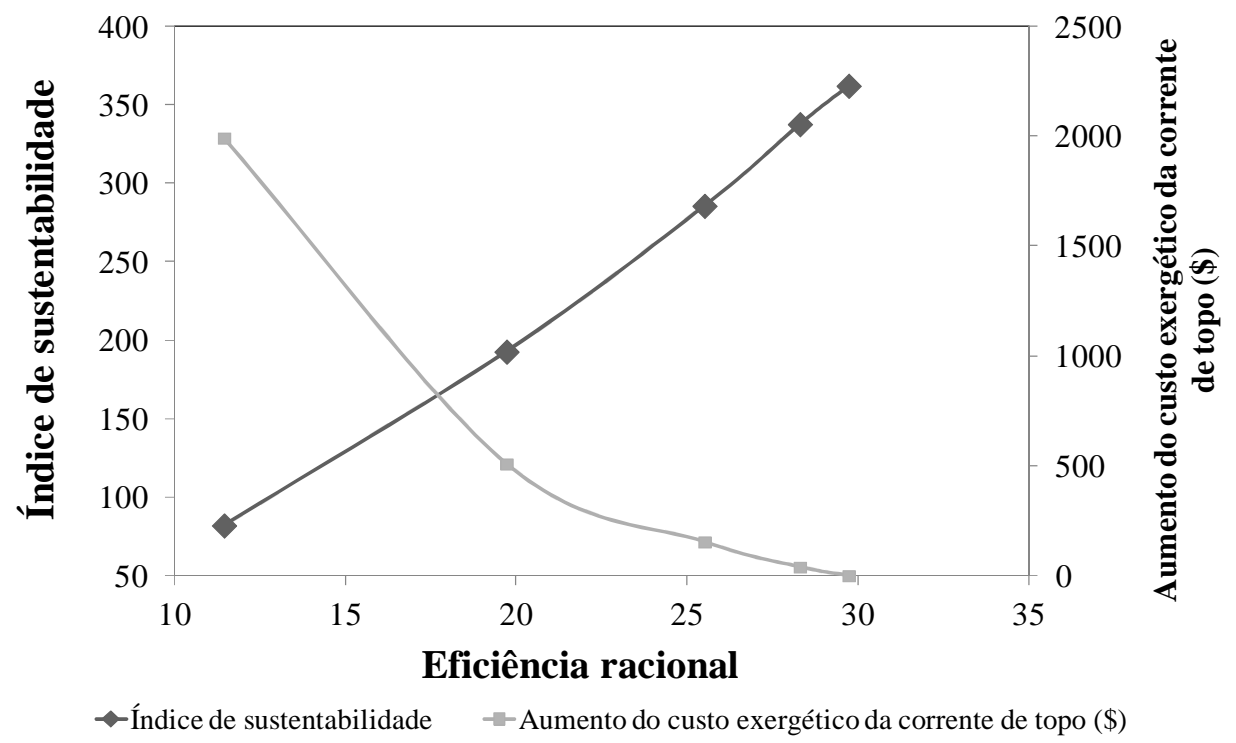

Figura 2 - Índice de sustentabilidade e impacto ambiental em relação ao cenário de razão de refluxo mínima da coluna despropanizadora, contra a eficiência racional da operação. 
No exemplo baseado em uma planta térmica simplificada, verificou-se que combustíveis com menor número de carbono são capazes de fornecer a mesma disponibilidade de energia que combustíveis de cadeias maiores, com quantidade inferior de emissão de dióxido de carbono, uma vez que, possuem um processo de combustão menos irreversível.

\section{CONCLUSÕES}

A exergia é baseada na segunda lei da termodinâmica e depende de um referencial, como por exemplo, o estado morto proposto por Szargut et al. (1988) que acarreta a limitação no número de espécies químicas que podem ser tratadas, pois apenas dez elementos químicos estão presentes. A metodologia proposta por Abdollahi-Demneh et al. (2011) contempla uma solução para espécies química contendo enxofre na composição.

$\mathrm{Na}$ aplicação desta metodologia em alguns exemplos, verificou-se que a irreversibilidade nos processos é proporcional aos gradientes de transporte de massa, calor, momento e de afinidade química. Observou-se, também, que a eficiência racional, equacionada por Ghannadzadeh et al. (2012), consegue descontar a exergia invariante. A implementação pode ser utilizada, por exemplo, para comparar em termos de exergia: processos criogênicos de separação de $\mathrm{N}_{2}$ e $\mathrm{CH}_{4}$ com uma, duas e três colunas; processos de captura de $\mathrm{CO}_{2}$, usando membranas não isotérmicas; e projeto de redes de trocadores de calor.

Enfim, a análise exergética é útil ao projeto de processos, sendo capaz de concatenar eficiência e sustentabilidade. Através de critérios de projeto de operações ou processos unitários, é possível efetuar modificações capazes de melhorar a eficiência e reduzir seu impacto ambiental, causado pela geração de desordem, mau aproveitamento dos recursos ou produção de rejeitos. O custo exergético contabiliza, portanto, o custo ambiental. A utilização deste parâmetro na formulação de custos econômicos pode ajudar a mensurar a viabilidade de tecnologias sustentáveis.

\section{AGRADECIMENTOS}

Os autores agradecem à CAPES, CNPq, FAPERJ e ANP.

\section{NOMENCLATURA}

$$
\begin{aligned}
& \text { Notação } \\
& v_{\gamma, \rho}
\end{aligned}
$$




$\begin{array}{llc} & \text { de referência } & \\ \mathrm{R} & \text { Constante dos gases } & 8314 \mathrm{~J} /(\mathrm{kgmol} . \mathrm{K}) \\ \mathrm{G}_{\mathrm{f}, \mathrm{i}} & \text { Energia de Gibbs molar de formação ideal do i-ésimo } & \mathrm{J} / \mathrm{kgmol} \\ & \begin{array}{l}\text { componente } \\ \mathrm{H}\end{array} & \mathrm{J} / \mathrm{kg} \\ \mathrm{S} & \text { Entalpia mássica } & \mathrm{J} /(\mathrm{kg} . \mathrm{K}) \\ \mathrm{B} & \text { Extropia mássica } & \mathrm{J} / \mathrm{kg} \\ \mathrm{Z}_{\mathrm{i}} & \text { Fração mássica } & - \\ \mathrm{MM} & \text { Massa molar do i-ésimo componente } & \mathrm{kg} / \mathrm{kgmol} \\ \mathrm{P} & \text { Pressão absoluta } & \mathrm{Pa} \\ \mathrm{X}_{0} & \text { Condição ambiente } & - \\ \mathrm{X}_{0 \mathrm{n}} & \text { Condição de referência do simulador } & - \\ \mathrm{P}_{\mathrm{ref}, \mathrm{i}} & \text { Pressão parcial do i-ésimo componente no estado } & \mathrm{Pa} \\ \mathrm{X}_{00} & \text { morto de referência } & - \\ \mathrm{T} & \text { Condição no estado morto de referência } & \mathrm{K} \\ \underline{\mathrm{Z}}_{i} & \text { Temperatura absoluta } & - \\ & \text { Vetor de frações molares dos componentes da i-ésima } & \end{array}$

\section{REFERÊNCIAS}

Abdollahi-Demneh, F.; Mohammad, A. M.; Mohammad, R. O.; Hossein, B. Calculating exergy in flowsheeting simulators: A HYSYS implementation. Energy, 23 Julho 2011. 36 5320-5327.

Ghannadzadeh, A.; Raphaele, T.; Olivier, B.; Philippe, B.; Pascal, F.; Xavier, J. General methodology for exergy balance in ProSimPlus process simulator. Energy, 10 Fevereiro 2012. in press.

Gharagheizi, F.; Mehrpooya, M. Prediction of standard chemical exergy by a three descriptors QSPR model. Energy conversion \& management, 4 junho 2007. 2453-2460.

Kotas, T. The exergy method of thermal plants analysis. London: Butterworths, 1985.

Lora, E. E. S.; Nascimento, M. A. R. Geração Termelétrica - Planejamento, Projeto e Operação. Rio de Janeiro: Interciência, 2004.

Rosen, M. A.; Dincer, I.; Kanoglu, M. Role of exergy in increasing efficiency and sustainability and reducing environmental impact. Energy Policy, 2008. 128-137.

Szargut, J.; Morris, D.; Steward, F. Exergy analysis of thermal, chemical, and metallurgical processes. Nova Iorque: Hemisphere Publishing Corporation, 1988.

Valero, A.; Lozano, M. A. Theory of the Exergetic Cost. Energy Vol. 18, Janeiro 1993. 939960.

Zanchini, E.; Terlizzese, T. Molar exergy and flow exergy of pure chemical fuels. Energy, 13 junho 2009. 1246-1259. 